\title{
Evaluating a trainee led induction day in psychiatry
}

\author{
BRIAN KIDD, Registrar in Psychiatry, Southern General Hospital, 1345 Govan Road, \\ Glasgow G51 4TF (Chairman, West of Scotland Psychiatric Trainees Committee); \\ RoBIN McGILP, Registrar in Psychiatry, Southern General Hospital; and \\ Cameron Stark, Registrar in Public Health, Argyll \& Clyde Health Board
}

\section{Background}

Many psychiatric training schemes hold induction days for their new junior doctors. This practice has now become a College recommendation and has frequently been a mandatory requirement for the approval of training schemes by the Royal College of Psychiatrists (Sims, 1990). Such days often concentrate on orientating the new trainee, looking in particular at facilities rather than the development of skills.

The West of Scotland Psychiatric Trainees' Committee (formerly the Strathclyde Psychiatric Club) has been organising a trainee led induction day since 1982. The aim has been to give some guidance about what is involved for junior doctors working in psychiatry, focusing on those areas which are seen as important by psychiatric trainees.

Various formats and topics for presentation have been used, a reflection of the feedback received from trainees attending previous meetings. The current format comprises a morning session during which five $\mathbf{4 5}$ minute presentations are given by members of the West of Scotland Psychiatric Trainees' Committee.

The subjects covered are: The Mental Health (Scotland) Act 1984; Assessment of Suicide Risk; Use of Drugs in Psychiatry; On-Call Duties and Assessment of the Violent Patient; General Practice Aspects of Psychiatry.

\section{The study}

A recent induction day was evaluated. Three weeks after it occurred an evaluation form was sent to all attenders. Although data were made anonymous after collection, questionnaires were numbered allowing a reminder to be sent two weeks later to those who had failed to reply. Basic demographic material was collected together with opinions on the topics, and on the quality of the presentations. Doctors were asked for an overall evaluation of the day and any ideas for improving future meetings.

\section{Findings}

Of the 30 questionnaires sent, $28(93 \%)$ were returned. Twenty-one $(75 \%)$ of the respondents were GP trainees. Five $(18 \%)$ were psychiatric trainees. The other two $(7 \%)$ were a physician rotating into psychiatry and an associated specialist wishing to increase his experience. Respondents had been qualified a median of two years (range $1-20$ years). Two had previous psychiatric experience, the longest time in psychiatry being ten months. All respondents felt that the day was at least useful with $24(86 \%)$ finding it very or extremely useful. No-one found the day to be of no use and $27(96 \%)$ stated they would recommend it to future juniors. In one case there was no answer to this question.

The topics seemed popular and in general the talks were well received. The most popular subject was the Mental Health Act, with all respondents finding it at least useful and $21(75 \%)$ finding it very useful. There were a few suggestions for improvement. The most frequent comment was the desire to see the session extended. Only one trainee stated that he had attended a similar induction in any specialty in the past.

\section{Comment}

To our knowledge this is the only induction day for junior doctors working in psychiatry organised by psychiatric trainees. The day is clearly acceptable and regarded as useful by those attending.

Most psychiatric training schemes already have a formal induction organised by the local tutor. Such occasions focus on the familiarisation of new doctors with local services and hospital arrangements as well as introducing trainees to paramedical staff and discussing modes of referral and appropriate use of paper work. The trainee led induction day is complementary, filling the gap between a traditional local induction day and the formal theoretical postgraduate training course. The aim is to supply practical advice on how to deal with events new trainees are likely to encounter from their first day in psychiatry. 
Our trainees' day is also an introduction to the Trainees' Committee.

\section{Practical considerations}

In organising such a day, it is essential to liaise with the local postgraduate advisers and hospital clinical tutors who can encourage their new trainees to attend. Details of it should be circulated well in advance (3-4 weeks before the day) and a reminder is useful in the preceding week. The timing is important and the day should not clash with hospital induction courses. We have found that the second week of an attachment is most suitable.

Choice of venue affects attendance. It must be accessible both to attenders and speakers and should be booked in advance. Venues can often be arranged free of charge. Speakers and topics should be chosen using feedback from previous presentations. It is important that the speakers are of high quality, have been briefed to avoid overlap and are well prepared.

We have found that by addressing such minor practical difficulties it is possible to organise an induction day which is well accepted locally and is valued by those who attend. We believe there is scope to establish similar trainee led induction days elsewhere and urge other trainees' groups to do so.

\section{Reference}

Sims, A. C. P. (1990) The approval of psychiatric training schemes in 1988. Psychiatric Bulletin, 14, 338-342.

\title{
From pulsating brains to paranoid psychoses
}

\author{
Gillian Doody, Registrar in Psychiatry, Lynebank Hospital, Halbeath Road, \\ Dunfermline, Fife, Scotland KY11 4VW
}

The recent technological explosion in the advancement of brain imaging, resulting in MRI, SPECT and PET scanning, has served to further blur the interfaces of neurology, neurosurgery and psychiatry. As proposed by Reynolds (1990), the traditional divergence of classical neurological science and psychiatry, originating from Cullen's description of 'neurosis', is currently changing to convergence and in many areas coalescing.

Inspired by an overwhelming sense of neurological inadequacy, consequent upon entering psychiatry immediately on completion of my pre-registration year in 1988, I decided to equip myself with a basic level of neurological competence in an attempt to function optimally as a psychiatrist in the years ahead.

Having negotiated a six month 'leave of absence' from my registrar rotation in Fife, I then set about scouring the job advertisements in the British Medical Journal. Initially I had intended applying for neurology SHO posts, but was disheartened by job descriptions indicating that on call "acute medical knowledge and experience would be required". Inexplicably, I then gravitated to the neurosurgical columns and finally applied for a post as SHO in a large teaching neurosciences centre. Once appointed it was with a sense of great trepidation that I embarked on what was to be a memorable and emotive six months.

To one accustomed to district general psychiatry, the intricacies of a tertiary referral centre are intrinsically alien. The transition from dealing primarily with GP-style ailments to being in the forefront of state of the art medicine was initially a terrifying experience for the yokel psychiatrist. Being previously limited to inserting an intravenous cannula perhaps twice a year, the ability to perform umpteen daily arterial blood gases, lumbar punctures and place central venous lines with any degree of competence initially seemed an impossible dream. However, with multiple failures, frequent embarrassment and considerable patience from sympathetic registrars, experience tentatively emerged from a trial and error protocol.

The first four months of the attachment were spent fulfilling ward duties with no obligation to attend theatre or out-patient clinics. Routine admissions were plentiful for elective vascular procedures, tumour resection, shunt insertion, investigative cerebral angiography and myelography. In addition an average of four emergency admissions each day ranging from head injuries to acute spinal cord compression could be expected. 